\title{
ZWEI SPRACHEN ZUM PREIS VON EINER
} $\mathbb{Z W E} S P R A C H I G \mathbb{E} \mathbb{E R Z} \mathbb{E} H \cup N G \mathbb{H E U T E}(\mathbb{T E} \mathbb{L} \mathbb{1})$

D folgende Abhandlung zum Thema Zweisprachigkeit fasst die wesentlichen Ideen und Aspekte der 1998 ins Spanische übersetzten und im Cambridge Verlag erschienenen Studie La Familia Bilingüe, Guia para padres von Edith Harding und Philip Riley (Originaltitel: "The bilingual familiy", 1986) zusammen.

Hiermit handelt es sich weder um eine Rezension lediglich einige in Klammern stehenden Aussagen haben kommentierenden Charakter -, sondern der Inhalt wird komprimiert und so originalgetreu wie möglich als dreiteilige Serie unter folgenden Themenblöcken wiedergegeben:

\section{1.) Das Phänomen Zweisprachigkeit \\ 2.) Die Entwicklung von Zweisprachigkeit (Heft 7 ) \\ 3.) Die Förderung von Zweisprachigkeit (Heft 8)}

prachiger Erziehung zu tun haben, ihre eigene - vielleicht ganz andere!? -Erfahrung darstellen können.

Wer ansonsten etwas zum Thema beitragen möchte, egal ob bestätigend, kritisierend, hinterfragend oder ironisierend, kann dies natürlich auch gerne in Form eines Leserbriefes tun. Jede Anmerkung zu irgendeinem der Aspekte ist willkommen und wird in einen "basisnahen" Erfahrungsbericht aus der andalusischen Praxis" aufgenommen.

Wir freuen uns auf eine rege Diskussion!

\section{"Zweisprachigkeit" - Was ist das eigentlich?}

Um die Problematik einer Definition von Zweisprachigkeit zu verdeutlichen, sei folgender frei erfundener, aber durchaus möglicher Dialog zwischen

Die Studie erhebt keinen Anspruch auf Wissenschaftlichkeit und Objektivität, sondern sie basiert auf den Beobachtungen und Erfahrungswerten von 16 Familien, die mehrheitlich der europäischen Mittelklasse angehören und ihre Kinder mit Erfolg zweisprachig erzogen haben. Diese Datenbasis wurde unter dem Aspekt der Nützlichkeit für andere Eltern

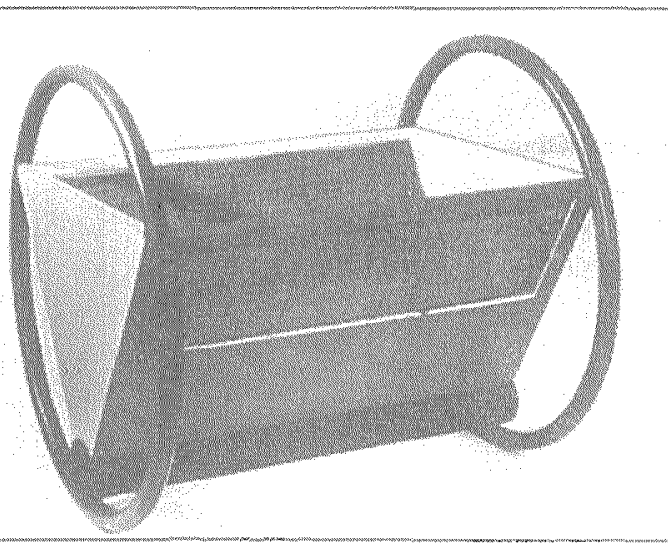
Mäxchen und Fritzchen vorangestellt:

"Sag mal, was bedeutet eigentlich

"Zweisprachigkeit"?"

"Ist doch sonnenklar: Wenn einer zwei Sprachen spricht!" "So wie Pierre?"

"Nee, der ist doch erst ein Jahr hier, der macht doch noch Fehler!"

"Aber nicht viele, und außerausgewertet und ist aus diesem Grund sicher- Peter Keler, cuna, 1922 dem hat der gar keinen Akzent, weil er als Kind lich für all jene interessant, die sich in einer hispano-germanischen Liaison befinden und den Schritt zur Familiengründung geplant oder bereits gewagt haben.

Die Vorstellung des Buches geschieht mit dem Hintergedanken, euch zur Mitarbeit an einem vierten Teil zum Thema "Zweisprachigkeit" aufzufordern: Im Anschluss an den dritten Teil in Heft 8 werden wir einen Fragebogen zu den wichtigsten Thesen des Buches veröffentlichen, damit alle Leser, die etwas mit zweisschon mal hier in Deutschland gelebt hat."

"Na ja, jedenfalls spricht der nicht perfekt Deutsch!"

"Aber die Smith macht überhaupt keine Fehler, wenn sie im Englischunterricht mal was auf Deutsch sagt. Also ist die zweisprachig, oder?"

"Hast du noch nicht gemerkt, dass die gar kein vernünftiges "r" sprechen kann? Und statt normal "Hallo" zu sagen, kriegt sie nur ein "Hallou" zustande, und überhaupt, es hört sich immer so an, als hätte sie 'ne heiße Kartoffel im Mund..." 
"Wie muss ein Zweisprachiger denn dann sprechen?" "Na, wenigstens so wie ich oder mein Vater..."

"So wie du? Dass ich nicht lache! Wenn ich mal was über meinen neuen Computer erzähle, dann guckst du dumm und verstehst nur Bahnhof,und neulich in der Autowerkstatt wusstest du noch nicht mal, was'ne Einspritzdüse ist. Wenn überhaupt jemand perfekt Deutsch spricht, dann mein Vater, der gibt Deutschunterricht an der Schule !"

"Alter Angeber! Wenn der so perfekt Deutsch kann, warum ist er dann wohl letzte Woche mit seinem komi schen Steuerbescheid zu meinem Vater ins Büro gekommen, häh? Natürlich, weil er den nicht verstanden hat, weil er inn nicht lesen konnte! Und als ich ihm dann gesagt habe, dass ich das ganz schön prickelnd finde, dass mein Daddy inm helfen muss, da hat er das auch nicht verstanden.!!!"

Das Problem liegt auf der Hand: Der allgemeinen Auffassung, dass Zweisprachigkeit bedeutet, zwei Sprachen perfekt zu beherrschen (oder vielleicht auch nur zu sprechen?!), muss entgegengehalten werden, dass es unmöglich ist zu bestimmen, wann dieses Perfektionsstadium erreicht ist. Niemand beherrscht sämtliche, in seiner Sprache verfügbaren Wörter, wir alle benutzen nur einen mehr oder weniger großen Teil derselben.

So spricht auch die zweisprachige Person lediglich Ausschnitte aus zwei Sprachen, die überdies selten übereinstimmen; wie z.B. sollten die Sprachkenntnisse eines Rechtsanwaltes, dessen Arbeitssprache Spanisch ist und der zu Hause Französisch spricht, miteinander verglichen werden?

Das Kriterium, eine Sprache wie ein Muttersprachler zu beherrschen, ist angesichts der enormen Unterschiede von Mitgliedern einer Sprachgemeinschaft bzgl. ihres Muttersprachenniveaus nicht haltbar. Festzuhalten ist lediglich Folgendes: Zweisprachigkeit kann nicht als ein klar abgrenzbares und eindeutig im Grad seiner Ausformung bestimmbares Phänomen betrachtet werden, sondern es muss stets nach seiner spezifischen graduellen Ausprägung gefragt werden; dabei kann sich eine außerordentliche Variationsbreite von Fall zu Fall ergeben.

Diese Betrachtungsweise liegt auch den meisten der zahlreichen und oft einander widersprechenden wissenschaftlichen Definitionen zugrunde, d.h., fast alle betonen den relativen Charakter von Zweisprachigkeit. So stellte L. Bloomfield schon 1933 fest:
"Die Zweisprachigkeit besteht in der Fähigkeit, zwei Sprachen wie ein Muttersprachler zu beherrschen. Selbstverständlich kann der Perfektionsgrad, der den eine Fremdsprache gut Sprechenden zu einem Zweisprachigen macht, nicht bestimmt werden: Die Unterscheidung ist relativ."

Bereitet die Definition des Phänomens Zweisprachigkeit auch einige Schwierigkeiten, so sieht es mit der Beschreibung seiner unterschiedlichen sozialen und individuellen Ausprägungen nicht ganz so kompliziert aus.

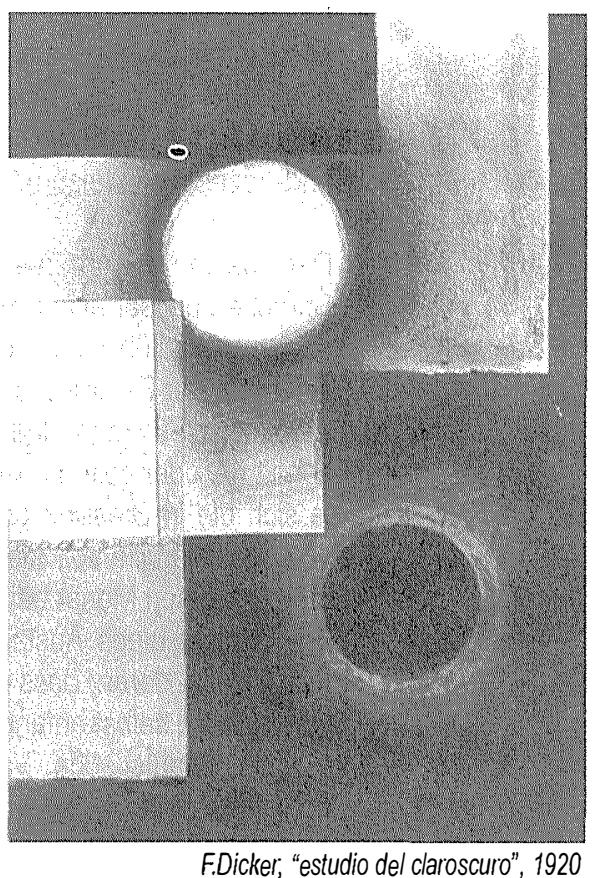

Auf soziologischer Ebene wird zwischen "elitärer" und "populärer Zweisprachigkeit" unterschieden, wobei erstere ein "Privileg der Mitglieder der gebildeten Mittelklasse in der Mehrheit aller Gesellschaften" darstellt (Paulston, 1975). Konkret sind damit z.B. Graduierte und Wissenschaftler gemeint, die ins Ausland gehen (und wohl auch die zahlreichen Deutschlehrer, die sich nach Andalusien verirrt haben?!) Dass es sich hierbei nicht um eine verschwindend geringe Minderheit handelt, belegen folgende Zahlen: Das Vereinigte Königreich schickt jährlich 80.000 Akademiker ins Ausland, und die Firma IBM hat 20.000 Beschäftigte in ganz Europa, davon $6.000 \mathrm{im}$ Ausland.

Demgegenüber bezieht sich die "populäre Zweisprachigkeit" auf Mitglieder der Arbeiterklasse und leitet sich von den "Bedingungen der ethnischen Gruppen, die gegen ihren Willen zweisprachig werden müssen, um zu überleben " ab (Tosi, 1985).

Die recht unterschiedliche Ausgangslage beider gesellschaftlicher Gruppen rechtfertigt eine solche Einteilung sicherlich, jedoch lässt sich die Vermutung, dass die in der zweisprachigen Erziehung auftretenden Probleme bei Mittelschichtkindern leicht gelöst werden können, nicht bestätigen. Auch hier sind Misserfolge und Abbrüche zu verzeichnen, wobei es in beiden Gruppen häufig die Mutter ist, die inre Muttersprache als Kom- 
munikationsinstrument innerhalb der Familie aufgibt und somit ihre linguistische Identität verliert.

Auf individueller Ebene lassen sich "asymmetrische" und "rezeptive_Zweisprachigkeit" unterscheiden:

Der "asymmetrisch Zweisprachige" spricht eine der Sprachen besser als er sie versteht, was auf die Tatsache zurückzuführen ist, dass er die Sprache lediglich innerhalb der Familie praktiziert hat und der Vielzahl an Akzenten, Stimmen und Sprachformen einer Gesellschaft nie oder selten ausgesetzt worden ist. (Man stelle sich die in Brasilien geborene Tochter einer hannoverschen Familie vor, die mit fünfzehn Jahren zum ersten Mal einen Monat bei einer bayrischen Familie, z.B. im Rahmen eines Austausches, verbringt! Es besteht kein Zweifel daran, dass sie von allen verstanden wird, während sie selbst anfangs kaum in der Lage sein dürfte, die linguistische Variante inrer Gastgeber zu entschlüsseln.)

Anzumerken ist an dieser Stelle, dass das Verstehensproblem von Dialekten, Sprechweisen und Akzent innerhalb eines Landes durchaus auch eine einsprachige Person betreffen kann, sich aber in beiden Fällen nach einer Phase der Anpassung von selbst löst.

Der "rezeptiv Zweisprachige" demgegenüber versteht eine der Sprachen, kann sie jedoch nicht sprechen. Diese Konstellation ist typisch für Kinder, von denen nur ein Elternteil eine Sprache Joost Schmidt. "Offset", revista spricht, die vom anderen de la Bauhaus dedicada al Elternteil nicht verstanden diseño del libro y la publicidad, wird, und für Inmigrantenfa1926

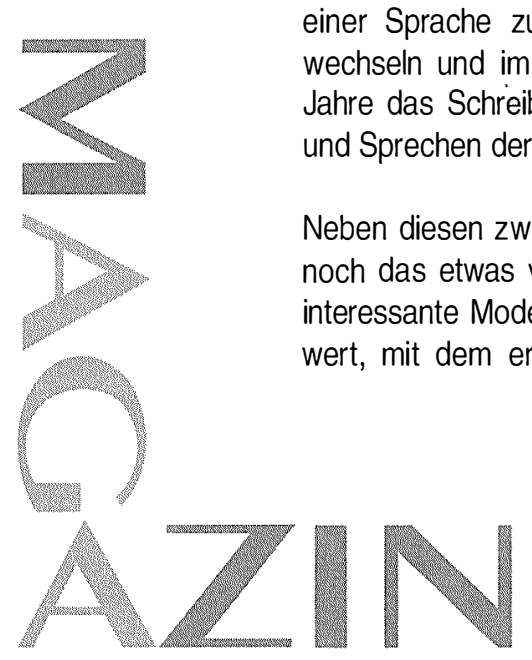

Struktur des Zweisprachigen vornimmt und zwischen "koordinierter", "zusammengesetzter" und "untergeordneter Zweisprachigkeit"unterscheidet:

Bei der "koordinierten Zweisprachigkeit" besitzt die Person zwei funktional unabhängige Systeme, d.h., sie benutzt zwei verschiedene linguistische Zeichen für jeden Referenten, welcher wiederum an eine andere Bedeutungseinheit gebunden ist. (Das spanische Wort "taverne" und das deutsche Wort "Kneipe" werden für zwei unterschiedliche Realitäten gebraucht.Vorstellbar ist eine Person, die sich lange sowohl in Spanien als auch in Deutschland aufgehalten hat und daher die feinen Unterschiede zwischen einer deutschen Kneipe und einer spanischen Taverne kennt.)

Die "zusammengesetzte Zweisprachigkeit" impliziert den Gebrauch zweier verschiedener Zeichen für eine miteinander verschmolzene Bedeutungseinheit. (Die Wörter "taverna" und "Kneipe" werden für die konstruierte Einheit "Takneiverpene" gebraucht. Vorstellbar ist eine Person, die die grundlegenden Unterschiede beider Lokalitäten niemals erfasst hat und in beiden

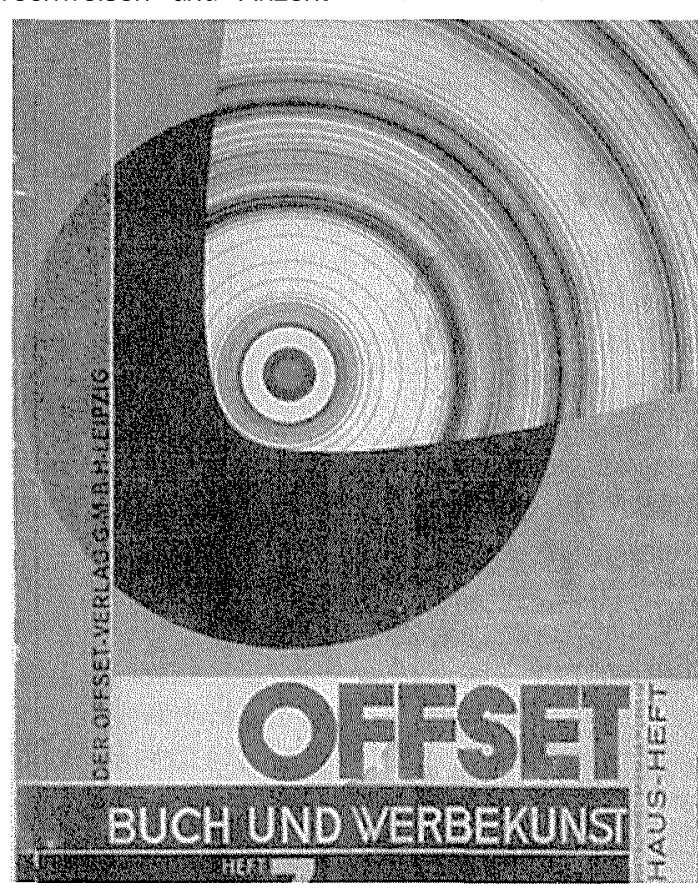
hauptsächlich das Gemeinsame, nämlich einen geselligen Ort zum Sitzen, Trinken und Plaudern, in einem Bild zusammenwürfelt. )

Bei der "untergeordneten Zweisprachigkeit" ist eine der Sprachen dominant; zwei verschiedene linguistische Zeichen beziehen sich auf nur eine Bedeutungseinheit. (Die Wörter "taverna" und "Kneipe" werden nur für die Realität TAVERNE bzw. nur für die Realität KNEIPE gebraucht. Vorstellbar ist eine Person, die zwar innerhalb der Familie Deutsch spricht, aber ausschließlich in Spanien gewohnt hat und demzufolge nie in den Genuss eines Kneipenbesuches kommen konnte, oder umgekehrt.)

Sicherlich lassen sich die meisten zweisprachigen Personen nicht schematisch in diese drei Kategorien einordnen, sondern sie liegen irgendwo "dazwischen" 
oder wechseln in Abhängigkeit von ihrer Tätigkeit und der Anwendung der Sprache von einer Kategorie zur anderen; zudem gibt es in den meisten Sprachen unzählige Wortpaare von nahezu identischer Bedeutung (z.B. ojo-Auge, papel-Papier, cuchara-Löffel), welche die o.g. Einteilung überflüssig erscheinen lassen.

Obwohl die Kategorien also kritisch hinterfragt werden müssen, können sie doch als kognitives Modell dienen, welches Aufschluss über eine ganz bestimmt Konstellation von familiärem und gesellschaftlichem Kontext gibt: So ist es wahrscheinlich, dass eine Person, die in den ersten zehn Jahren einsprachig in der Familie aufgewachsen ist und dann mit einem anderssprachigen außerfamiliären Kontext konfrontiert wird, zum Vertreter der koordinierten Zweisprachigkeit wird, in der zwei Systeme unabhängig voneinander existieren.

Dagegen wird eine in einem vollkommen zweisprachigen familiären Kontext aufgewachsene Person eine Tendenz zur zusammengesetzten Zweisprachigkeit aufweisen, in der sich die Bedeutungseinheiten vermischen und die linguistischen Zeichen beider Sprachen gleichermaßen dafür benutzt werden.

Ein Kind schließlich, das in einem Haushalt aufwächst, wo zwei Sprachen innerhalb des gleichen Systems von Referenten benutzt werden, entwickelt eine untergeordnete Zweisprachigkeit.

Nach diesem Exkurs durch mögliche und unmögliche Definitions- und Beschreibungsversuche stellt sich nun die Frage nach dem, was die Besonderheit des Phänomens Zweisprachigkeit ausmacht. (Für einige werden hier recht ernüchternde Tatsachen aufgedeckt!)

\section{Zweisprachigkeit - ein ganz normales Phänomen!}

Über die Hälfte der Weltbevölkerung ist zweisprachig! Diese Feststellung mag den Europäer, der Zweisprachigkeit als etwas ganz besonderes betrachtet, überraschen, da sie der allgemeinen Vorstellung von der Beziehung "eine Sprache - ein Individuum - eine Nation" widerspricht. In Anbetracht der Tatsache allerdings, dass weltweit 3000-5000 Sprachen, aber nur ca. 150 Länder existieren, wird es vollkommen offensichtlich, dass Zweisprachigkeit im Vergleich zur Einsprachigkeit das "normalere" Phänomen darstellt.
Selbstverständlich gibt es enorme Unterschiede bezüglich der Verbreitung der einzelnen Sprachen: So spricht fast ein Viertel der Weltbevölkerung Chinesisch -ob es sich hier um eine Sprache handelt, sei dahingestellt-, während die Sprechergemeinschaft des schottischen Gälisch verschwindend gering ist.

Auf nationaler Ebene sind offiziell einsprachige, zweisprachige und mehrsprachige Gesellschaften zu unterscheiden, wobei der offizielle Status jedoch nichts über die reale Verteilung von Zweisprachigkeit aussagt. Im Gegenteil - diese ist in offiziell einsprachigen Nationen wie z.B.Tansania oft höher als in offiziell zweisprachigen Nationen wie z.B. Kanada. So sprechen 90\% der Bevölkerung Tansanias gewöhnlich mindestens zwei der etlichen Landessprachen, während nur $13 \%$ der kanadischen Einwohner gleichermaßen Französisch und Englisch benutzen. Ähnlich sieht es in der mehrsprachigen Schweiz aus, wo die offiziellen Sprachen Deutsch, Französisch und Italienisch kaum von einem Schweizer gleichzeitig beherrscht werden.

Eine andere Variante stellt die mehrsprachige indische Gesellschaft dar, deren Verfassung insgesamt vierzehn Sprachen anerkennt, von denen Englisch und Hindi die offiziellen sind; nichtsdestotrotz benutzen nur 10\% der Bevölkerung mehr als eine Sprache.

So können theoretisch zweisprachige Gesellschaften existieren, in denen alle Individuen einsprachig sind, wie auch in einer offiziell einsprachigen Gesellschaft alle Mitglieder zweisprachig sein könnten. Diese Feststel lung verdeutlicht die Notwendigkeit einer Trennung der gesellschaftlichen von der individuellen Ebenen, welche zur grundsätzlichen Unterscheidung zwischen gesellschaftlicher und individueller Zweisprachigkeit führt.

Soll z.B. - wie es das Ziel dieser Abhandlung ist - die Bedeutung von Erziehung in zweisprachigen Kontexten untersucht werden, so muss von der individuellen Zweisprachigkeit ausgegangen werden. 
Eine weitere aufschlussreiche Frage bei der Klärung des Phänomens Zweisprachigkeit ist diejenige nach dem Unterschied zwischen Sprache und Dialekt: Einer verbreiteten Ansicht zufolge ist jemand zweisprachig, wenn er zwei Sprachen spricht, dagegen aber einsprachig, wenn er - wie ein Großteil der Weltbevölkerung eine Sprache und einen Dialekt beherrscht.

Vom linguistischen Standpunkt aus ist aber keine klare Grenze zwischen Sprache und Dialekt zu ziehen, was bereits aus der diffusen Angabe von 3000-5000 existierenden Sprachen hervorgeht. Als "Sprachen" bezeichnete Gruppen wie z.B. die skandinavischen Sprachen können mehr Ähnlichkeit miteinander haben als die Dialekte einer Sprache wie Niederbayrisch und Hamburger Platt.

Die Definitionen von "Sprache" und "Dialekt" sind politischen und gesellschaftlichen Ursprungs und haben willkürlichen Charakter. Als "Sprache" bezeichnet man einen offiziell anerkannten Dialekt, welcher in den oberen gesellschaftlichen Schichten, in offiziellen Bildungsund Ausbildungsstätten, in der Verwaltung, in Massenmedien etc. benutzt wird. Der Gebrauch des Begriffes "Norwegisch" verweist nicht auf die Existenz einer linguistischen, sondern auf die einer politischen Einheit. Konflikte bezüglich der Anerkennung von Dialekten als Sprache sind stets politischer Natur; man hat "Sprache" ganz unwissenschaftlich, aber dafür umso ausdrucksstärker, auch als "Dialekt mit eigenem Heer" bezeichnet.

Auf der Grundlage dieser Sprachdefinition läßt sich eine außerordentlich interessante Schlussfolgerung ziehen:

Vom linguistischen Standpunkt aus besteht kein grundsätzlicher Unterschied zwischen einer Dialekt und Hochsprache und einer zwei Sprachen sprechenden Person, d.h. die Unterscheidung zwischen Ein- und Zweisprachigkeit wird in ihren Grenzen aufgeweicht.

Das bedeutet, dass der schwäbische Ministerpräsident, der nach einer harten Parlamentsdebatte auf Hochdeutsch nach Hause kommt und mit seiner Frau auf Schwäbisch übers Essen plaudert, im Prinzip nichts anderes macht als die Mutter, die ihr Kind auf Deutsch zum Essen ruft und sich dann bei ihrem Ehemann auf Spanisch über die lästige Hausarbeit beschwert.
Genau wie eine Hochsprache und Dialekt beherrschende Person die gleichen Fähigkeiten zeigt und die gleichen Mechanismen anwendet wie ein Zweisprachiger, so ist dies auch bei all denjenigen der Fall, die in ihrer Muttersprache verschiedene Stilebenen unterscheiden und korrekt gebrauchen können. Mit anderen Worten:

"Wir können unfehlbar davon ausgehen, dass wir alle zweisprachig sind: Jedes Mal, wenn wir aus zwei unterschiedlichen Formen, die das Gleiche ausdrücken, eine auswählen, z.B. "Hola,Diego, cómo estás"?" statt "Buenos días, Señor López", machen wir genau das Gleiche wie eine zweisprachige Person, die eine der beiden Sprachen wählt." (Harding/Riley, S.28).

Nachdem der Mythos vom "exotischen Zweisprachigen" nun Schritt für Schritt abgebaut worden ist, kommen wir

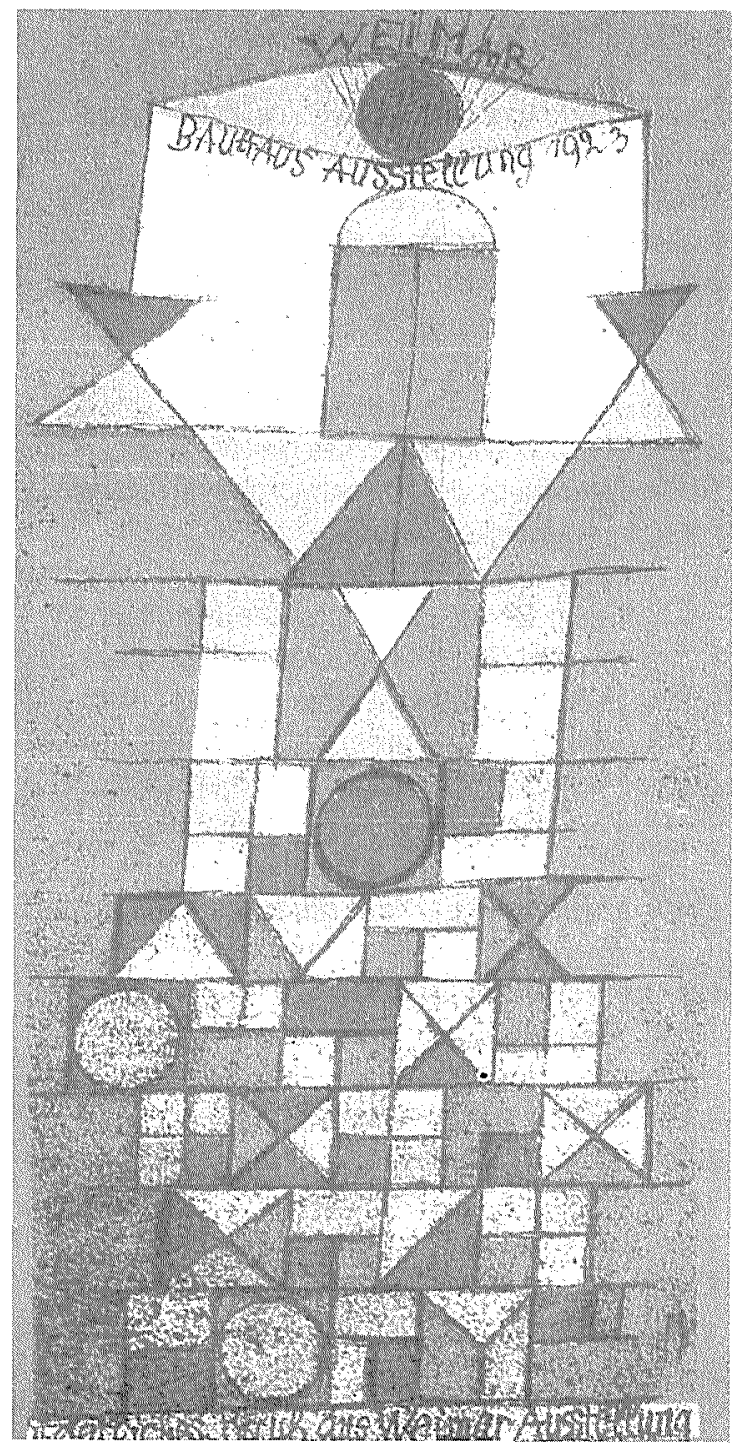

Paul Klee, 1923

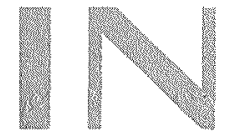


zu einer weiteren spektakulären Frage:

Sind Zweisprachige intelligenter?

Auch hier wieder eine Enttäuschung: Es existieren bisher keine gesicherten Ergebnisse bezüglich eines Zusammenhanges von Zweisprachigkeit und Intelligenz - wie immer diese auch definiert sein mag!

Genauso wenig ist eine Beziehung zwischen Zweisprachigkeit und linguistischen Fähigkeiten, kognitivem Funktionieren, emotionaler Anpassung und ähnlichen Faktoren festzustellen.

Unterschiedliche Studien kamen im Laufe der letzten Jahrzehnte zu unterschiedlichsten Ergebnissen: Mal wurde Zweisprachigkeit als negativ, mal als positiv bezeichnet, jedoch stellte sich letzlich heraus, das keine der Studien als vertrauenswürdig anzusehen war, da grobe methodologische Fehler begangen worden waren. So hatten hauptsächlich sozio-ökonomische Faktoren wie die Zugehörigkeit der Befragten zu einer bestimmten sozialen Schicht die Ergebnisse völlig in eine bestimmte Richtung hin verzerrt.

Zum Beispiel bezog sich die Studie von Saer (1928) auf Englisch und Gälisch sprechende, aus einer ländlichen Gegend kommende Kinder mit einem niedrigeren Intelligenzquotienten als die einsprachigen Kinder; außerdem zog man den Grad an Zweisprachigkeit nicht in Betracht. Da die Prüfung vorwiegend verbalen Charakter besaß, erzielten die Einsprachigen wesentlich bessere Ergebnisse und rechtfertigten so die Annahme, Zweisprachigkeit sei etwas Schlechtes.

Einige Jahre später zeigte Arsenian (1937) anhand einer Studie New Yorker Kinder, dass beide Gruppen ähnliche Ergebnisse erzielten, wenn die Art der Prüfung überwiegend nonverbalen Charakter hat.

Zu einem ganz anderen Resultat kamen Peal und Lambert (1962) bei der Untersuchung zweisprachiger Kinder in Montreal: Diese schnitten durchweg besser ab als inre einsprachigen Kameraden, was auf ihre mentale Flexibilität und ihre kognitiven Fähigkeiten zurückgeführt wurde. Zwar zeigten die Autoren Vorsicht bei der Herstellung einer kausalen Beziehung von Zweisprachigkeit und Intelligenz, indem sie die Frage "Fördert das eine das andere oder umgekehrt?" unbeantwortet ließen, aber sie hatten dennoch übersehen, dass sie die Erhebung bei Kindern aus Familien mit einem ziemlich hohen sozio-ökonomischen Niveau durchgeführt hatten und es sich außerdem um eine Region in Kanada handelte, wo beide Sprachen ein hohes Prestige genießen und dementsprechend gefördert werden.

Zusammenfassend (und etwas resigniert?!) stellt McLaughlin (1978) fest: "Das einzige durch wissenschaftliche Untersuchungen belegte Ergebnis ist, dass die Beherrschung einer zweiten Sprache signifikant ist, wenn das Kind sich einer Prüfung in dieser Sprache unterzieht, was nicht weiter erstaunlich ist."

Statt sich weiterhin mit dem Problem "intelligenter oder nicht" abzumühen, wendet man sich gegenwärtig eher der Frage nach den Unterschieden zwischen Ein- und Zweisprachigen zu:

Abgesehen von der unbestrittenen Tatsache, dass das Beherrschen einer zweiten Sprache unmittelbaren praktischen (z.B. beim Reisen) und sozialen (z.B. Anerkennung durch Freunde) Wert hat, besitzt die betroffene Person unbewußt Kenntnisse bezüglich vieler Aspekte der Sprache, die für den Einsprachigen beim Erlernen einer Zweitsprache ein Problem darstellen. So z.B. werden grammatische und phonetische Normen sowie lexikalische Besonderheiten nicht skeptisch hinterfragt und misstrauisch beäugt, sondern spontan assimiliert. (Die Deutschen zerhacken nun mal einige Verbformen und verdammen das erste Stückchen ans Satzende - na und?).

Der Zweisprachige hat ein Bewusstsein bezüglich der Willkürlichkeit der Beziehung von Sprache und Objekt, auf das sich die Sprache bezieht. Durch diese Abstraktionsübung wird sein Abstraktionsvermögen geschult, was schon im frühen Alter zur Flexibilität und Offenheit im Denken führt.

Diese Fähigkeiten sind unter anderem auf das Denken in zwei Sprachen zurückzuführen - ein Prozess, dem sich der Zweisprachige oft selbst nicht bewusstist. Zwar gibt es auch prä- und nonverbale Denkprozesse, die sogenannten "inneren Monologe" aber die werden in einer Sprache nach Wahl verbalisiert. Mit der Fähigkeit des zweisprachigen Denkens als eine Art "lateralen Denkens" verfügt der Zweisprachige über ein zusätzliches Instrument zur kreativen Gedankenarbeit und zur Lösung von Problemen.

Weiter kann als erwiesen angesehen werden, dass Zweisprachigkeit zum Fremdsprachenlernen befähigt: 
Nicht nur der Akzent des Zweisprachigen ist beim Erlernen einer weiteren Sprache im allgemeinen besonders gut, da ja aus zwei verschiedenen Repertoires geschöpft werden kann, sondern insgesamt herrschen eine besonders positive Grundhaltung und ein großes Selbstvertrauen in die eigenen Fähigkeiten hinsichtlich des Erlernens von Fremdsprachen vor.

Schließlich sei im Zusammenhang mit Zweisprachigkeit auch die Tendenz zum "Bikulturalismus", welcher ein geschärftes Bewusstsein für unterschiedliche kulturelle und gesellschaftliche Phänomene impliziert,erwähnt. Dies beruht auf der Kapazität, zwei Kulturen in ihren Aspekten zu vergleichen und zu relativieren sowie die Verhaltensmodelle einer Gesellschaft aus einem anderen Blickwinkel zu betrachten. Die Trennung des Kulturspezifischen vom Universellen erlaubt eine gewisse Unabhängigkeit der Beobachterposition und schafft die für die Bewusstwerdung diverser gesellschaftlicher Realitäten notwendige Distanz.

Der "Bikulturalismus" fördert beim Individuum die Resistenz und die Verarbeitungskapazität von Konflikten und Widersprüchen; komplexe Zusammenhänge werden als Ganzes erfasst und nicht auf einfache Verstehensmodelle reduziert, was insgesamt das Fundament für Toleranz gegenüber "dem Anderen" legt. (Ganz wichtig z.B. im Kampf gegen Rassismus und Diskriminierung von Minderheiten!) So werden wir letztendlich für die frustierende Nachricht, dass unsere mühsam zweisprachig erzogenen Kinder nicht automatisch einen höheren IQ aufweisen, doch mit einer Reihe ansprechender Merkmale entlohnt!

Nachdem wir nun das Phänomen Zweisprachigkeit im Groben und im Feinen beleuchtet haben, wollen wir uns im folgenden Kapitel der Frage nach dem individuellen Entwicklungsprozess der Zweisprachigkeit widmen.

C.S. 10

\section{Literaturverzeichnis}

ARSENIAN, S. (1937) Bilinguism and mental development. Teacher's College Contribution to Education, 712. Nueva York: University of Colombia.

BLoomfield, L. (1933) Language. Nueva York: Holt , Tinihart and Winston.

McLaUghuIN, B. (1978), SecondLanguage Acquisition in Childhood. Hillsdale, N.Y.: Lawrence Erlbaum.

PAULSTON, C.B. (1975) "Ethnic relations and bilingual education: acounting for contradictory data", Working papers on Bilingualism, 6, S.368-401.

Peal, E. and Lambert, W.E. (1962) "Relation of bilinguism to intelligence", Psychological Monographs, 76, S.1-23.

SAER,O.J. (1923) "The effects of bilinguism on intelligence". British Journal of Psychology, 14, S.25-38.

Tosı, A. (1982) "Mother tongue teaching for the children of migrants". In V. Kinsella (ed.) Surveys, 1, Cambridge Language Teaching Surveys. Cambridge University Press.

WEINREICH, U. (1953) Languages in Contact, La Haya:Mouton.

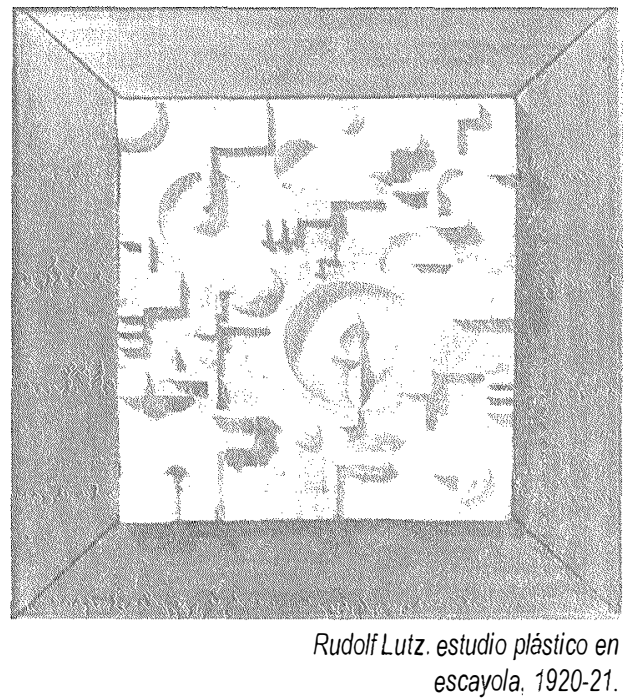


Sprechen Sie Deutsch?

\section{SOETHE NSTITUT}

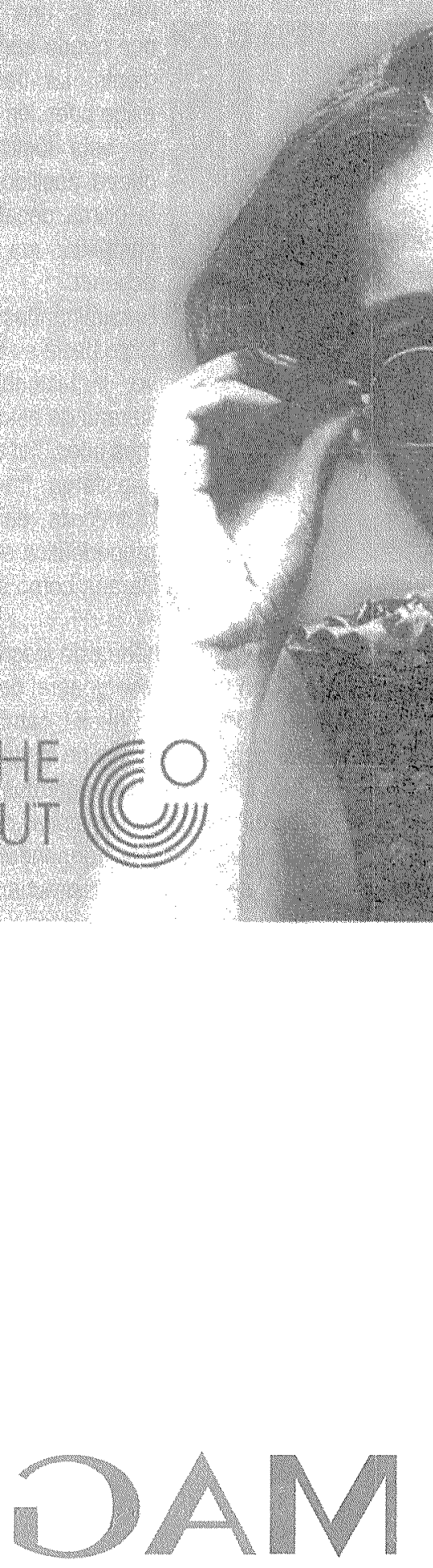

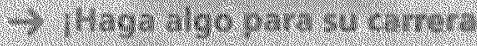

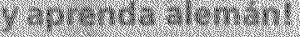

(1)

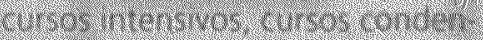

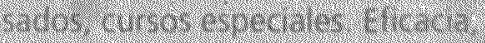

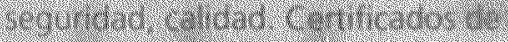

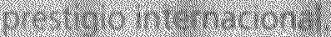

Kurse in Deutschland:

Goethe Institut, Postfach 190419, 80604 München

Tel. 0049-89-15921-200.

Fax 0049-89-15921-202,

e-mail:esb@goethe.de

www.goethe.de/deutschland

\section{Goethe Madrid:}

Tel. $91-3913966$, Fax 91-391 3945 , e-mail: gi@madrid.goethe.org www.goethe/madrid
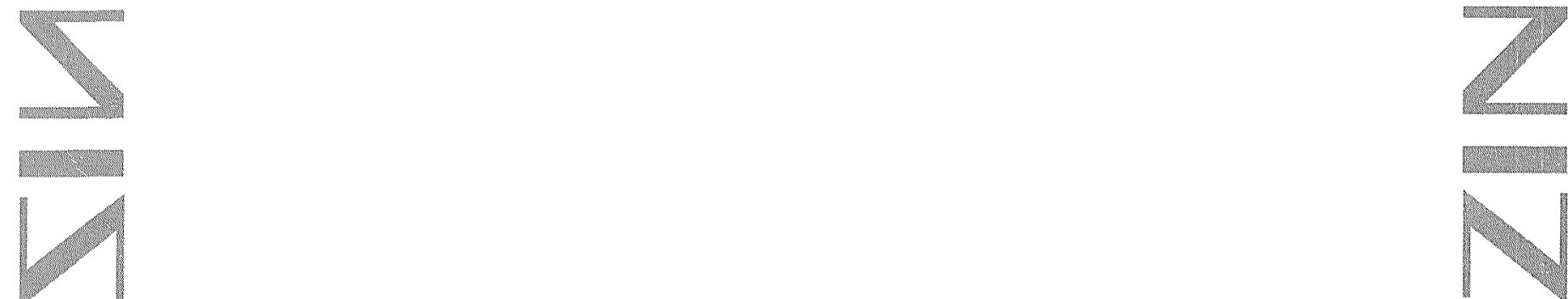